\title{
Psychometric properties of observational tools for identifying motor difficulties - a systematic review
}

\author{
P. Asunta ${ }^{{ }^{*}}\left(\mathbb{D}, \mathrm{H}\right.$. Viholainen ${ }^{2}$, T. Ahonen ${ }^{2}$ and P. Rintala ${ }^{1}$
}

\begin{abstract}
Background: Early identification of children with motor difficulties, such as developmental coordination disorder (DCD), is essential. At present only a fraction of children with DCD are identified. The purpose of the study was to systematically review the literature from 1994 to 2017 on observational screening tools and to evaluate the validity, reliability and usability of the questionnaires used.

Methods: The review of the literature was conducted to synthesize the data from five electronic databases for children aged 6-12 years. The following databases were searched: Academic search Elite (EBSCO), ERIC (ProQuest), MEDLINE (Ovid), PsycINFO (ProQuest), and SPORTDiscus with Full Text (EBSCO). The studies meeting our inclusion criteria were analyzed to assess the psychometric properties and feasibility of the measures.

Results: The literature search retrieved 1907 potentially relevant publications. The final number of studies that met the inclusion criteria of our systematic review was 45. There were 11 questionnaires for parents, teachers and children. None of the questionnaires was valid for population-based screening as the only measurement tool.

Conclusions: There are many challenges in using initial screening tools to identify children with motor difficulties. Nevertheless, many promising questionnaires are being developed that can provide information on functional skills and limitations across a variety of tasks and settings in the daily lives of children with DCD. The review provides much needed information about the current scales used in many clinical, educational and research settings. Implications for assessing psychometric properties of the developed questionnaires and further research are discussed.
\end{abstract}

Trial registration: PROSPERO, CRD42018087532.

Keywords: Developmental coordination disorder, Questionnaire, Assessment, Psychometric properties

\section{Introduction}

Developmental coordination disorder (DCD) has been discussed for 20 years, at present only a fraction of children with DCD are identified [1]. DCD is still poorly understood by many healthcare and education professionals [2], although, DCD affects 5-6\% of school-age children. It is characterized by a major impairment of motor coordination and typically has a significant negative impact on the performance of everyday activities or academic achievement [3].

\footnotetext{
*Correspondence: piritta.asunta@gmail.com

${ }^{1}$ Faculty of Sport and Health Science, University of Jyväskylä, P.O. Box 35,

Fl-40014 Jyväskylä, Finland

Full list of author information is available at the end of the article
}

Early assessment and identification of children at risk for DCD are important in order to avoid these secondary physical, cognitive, language, and social-emotional manifestations of the disorder $[4,5]$. There is considerable evidence that difficulties to acquire and execute motor skills can lead to secondary problems, such as poor selfesteem and other psychosocial issues [6,7] and physical health problems [8]. Furthermore, DCD is commonly associated with other developmental disorders [9], such as attention deficit/hyperactivity disorder (ADHD) [10, 11], learning disabilities such as dyslexia and specific language impairment (SLI) [12], and autism and associated psychosocial impairments [13, 14]. However, identification of DCD is difficult especially in school context

(c) The Author(s). 2019 Open Access This article is distributed under the terms of the Creative Commons Attribution 4.0 International License (http://creativecommons.org/licenses/by/4.0/), which permits unrestricted use, distribution, and reproduction in any medium, provided you give appropriate credit to the original author(s) and the source, provide a link to the Creative Commons license, and indicate if changes were made. The Creative Commons Public Domain Dedication waiver (http://creativecommons.org/publicdomain/zero/1.0/) applies to the data made available in this article, unless otherwise stated. 
because of DCD's heterogeneity in severity and comorbidity and its appearance in the area of fine and/or gross motor skills.

Up to now, the greatest emphasis has been on diagnostic screening. Especially, in the field of DCD, the goal has been to identify those with movement difficulties [15]. Along with home, the school environment is a place where children spend a lot of time; therefore, teachers perceive the child's performance in everyday activities and academic learning, which is one of the diagnostic criteria of DCD [3]. There are also studies that emphasize the importance of involving teachers in DCD screening $[16,17]$. Moreover, providing teachers with an easy-to-use method for identifying problems in motor learning could support them in their quest to enhance all children's motor learning. Practical tools for teachers are needed, because it has been found that teachers are more likely to recognize motor problems if nondisruptive behavior is present [18]. This is alarming, since we know the comorbidity with DCD and other psychosocial difficulties [11, 19, 20]. However, we did not limit our interest strictly to school teachers, as our focus of screening tools was context free.

Few observational tools for teachers, parents, children and nurses to identify children with motor learning problems have been developed. Those checklist-type tools have been extensively used both in research and non-research settings in the field of DCD [15]. Barnett [15] has highlighted that further studies are needed to establish the utility of each of these instruments to accurately identify children with DCD.

Therefore, we were interested in evaluating which of the developed questionnaires could be feasible, valid, and reliable for further development as cultural adaptations, which enable exchange of information and facilitate collaboration between countries and which furthermore are cheap and fast [21]. There being no replicable studies available, we conducted our own comprehensive systematic review. The specific aim of the systematic review was: (1) to investigate the questionnaire-based (paper-pencil) identification tools for psychometric properties and (2) to describe the usability in identifying motor difficulties in primary school-age children (6-12-years old) in different environments. We use words 'identifying' and 'screening' as synonyms, though there is a small difference between them. Identifying is more suitable in educational approach and screening in medical and research settings.

Currently, there is no gold standard tool to assess children with DCD [7]. Many instruments are available to investigate motor ability in children [5]. In order to measure movement competence, a wider range of test batteries is recommended [22], as well as a multi-stage approach. In a multi-stage approach a preliminary screening is usually carried out by questionnaire-based observational tools, which provide economical and effective first-step assessment [23], and the results can be followed or confirmed by standardized tests [24-26].

Despite the advisability of early assessment and identification $[16,24,26]$ and the development of many screening instruments, there are no gold standard observational tools available either. Indeed, although the disorder is so common, basic information about feasible and valid observational questionnaires for identifying problems in motor skill acquisition, which is one the most important criteria of DCD, is still lacking. Furthermore, it is uncertain who might be the most reliable and valid person to make qualitative observations: teacher, parent, or the child him/herself. Green and Wilson [27] have suggested that parents and children can assist in the screening process, because their judgments about movement difficulties are valid. However, it has been postulated that parents and teachers often over-refer the problems [28]. In contrast, parents' information is arguably essential to determine, whether the child's motor impairment is actually impacting on everyday activity like self-care skills (e.g., washing and dressing), Along with home, the school environment is a place where children spend a lot of time; therefore, teachers perceive the child's performance in everyday activities and academic learning. The screening instruments in home and school settings can be usefully applied to the assessment of criterion $\mathrm{B}$, to obtain information on the range of everyday life skills (ADL) that the child finds difficult, which is one of the diagnostic criteria of DCD $[3,29]$.

Psychometric properties refer to the validity and reliability of the measurement tool. Before being able to state that a questionnaire has excellent psychometric properties, meaning a scale is both reliable and valid, it must be evaluated extensively [30].

Information on usability can be gathered and described on both the literature and the experience of people using experts, user interviews and statistics. For practicability the following features can be evaluated: price, availability / usage restrictions, education needed, time requirements, ambiguity and ease of interpretation of results (including availability of reference values).

Many studies have underlined the challenges of using initial observational screening tools to identify children with DCD in population-based samples [31, 32]. In clinical studies, the concurrent validity (sensitivity and specificity rates) are somewhat better than in populationbased studies, but they are still not acceptable [32] Screening tools have been shown to have the ability to identify true cases of DCD (sensitivity) when it is present but infrequently the ability to exclude DCD when it is absent, in other words correctly to identify children without DCD (specificity) [29]. However, good sensitivity $(>80 \%)$ is more preferable in population screening than 
high specificity (>90\%) in order to identify all children at risk [25, 32]. Sensitivity has been found to be generally weaker in population based data sets than in clinical-referred samples [32].

\section{Method}

Protocol

Details of the protocol for this systematic literature review was registered with the international database of prospectively registered systematic reviews, PROSPERO, and given the registration number CRD42018087532 (can be retrieved at www.crd.york.ac.uk/PROSPERO/display_record. asp?ID=CRD42018087532.). Our search strategy utilized and combined the following main areas of keywords and synonyms. The terms were chosen according to the study questions and from those found in the literature on DCD studies, as indicated in our preliminary search. We had two groups of words (A and B). The words / key terms in group A were synonyms for DCD: clumsy children, developmental coordination disorder (DCD), probable DCD, motor skills disorder, minimal brain dysfunction, dyspraxia, movement disorders, motor problems, motor difficulties, motor learning difficulties, incoordination, and motor delay. The terms in group B described observational measurement tool: screening, screening tool, questionnaire, and checklist. To be considered for inclusion in the review, the title or abstract of the study had to contain at least one term from both of the groups (A and B).

The following five electronic databases were searched for the review: Academic search Elite (EBSCO), ERIC (ProQuest), MEDLINE (Ovid), PsycINFO (ProQuest), and SPORTDiscus with Full Text (EBSCO). In addition, we conducted searches in Google Scholar to retrieve supplementary information. Information was also sought manually, for example among the references in the identified publications, and EACD recommendations [33] were reviewed. Colleagues in the field were also consulted.

The search, which was designed to be inclusive and accurate, followed research guidelines [34]. Database-controlled vocabulary (Thesaurus) was used whenever possible. The terms used were tailored for each database. Full details of the searches can be found in Additional file 1.

Studies were included if the following criteria were met: (1) published in a peer-reviewed journal; (2) published between 1994 and 2017; (3) containing at least one term from both keyword groups (A and B); (4) relating to children aged six to 12 years (or mean $\geq 6$ ); (5) English language; (6) observational questionnaire (paperpencil instrument).

Studies were excluded: (1) related only to clinical assessment screening tests, because our interest was in finding questionnaire-based, short, and easy-to-use methods for identifying problems in motor learning; (2) they fell outside the diagnostic exclusion criteria of DCD according to DSM-V [3], such as neurologic disorders, other specific learning disabilities, or intellectual disability.

In the first stage of the screening process, the studies were considered based on their titles and abstracts. The second stage was approval on the basis of the full text. Manually found articles were included in the full text screening stage. Two independent reviewers (PA and $\mathrm{HV}$ at the level of titles and abstracts, and PA and PR at full text level) screened and selected articles at each stage of the selection process and checked the differences between the accepted titles, abstracts, and full texts. Where there was disagreement, reviewers discussed the issue until they reached a consensus. Consistency between the two authors before consensus discussions varied from $94 \%$ at the abstract level to $92 \%$ at the full text level.

\section{Evidence synthesis and quality assessment}

Studies that were selected, having met our inclusion criteria, were reviewed to collect descriptive psychometric information. They were divided according to their terms of measurement, aim, age, scope/population, and psychometric properties (see Additional file 2). The quality of the selected articles was evaluated based on the Grading of Recommendations Assessment, Development, and Evaluation (GRADE) methodology. GRADE classifies the quality of evidence as high, moderate, low, or very low [35-37]. Since this method is primarily intended to evaluate interventions and diagnostic tools, we modified the GRADE criteria (see Table 1). For example randomized trials without important limitations provide high quality evidence and observational studies without special strengths or important limitations provide low quality evidence. Factors that reduce or increase the level of evidence 1 or 2 levels, are described in Table 1. For instance, if the sample selection is well described, sample size is large or very good representativeness of the population and we think that the data has been analyzed with relevant statistical tests and quality of results are good, it is possible to reach the highest level of evidence.

In a modern view "validity is ensuring the appropriateness of an inference or decision made from measurement" [38]. Further, validity can be thought to be a characteristic of the inferences made based on the results obtained using the questionnaire or measurement tool [39]. Continuous validity evaluation of the developed methods is essential and should be viewed as a unified concept [38, 39].

We looked for different aspects of empirical validity evidence, including concurrent, predictive, construct, known group/discriminative, convergent, cross-cultural, and face validity. Concurrent validity relates to how well a measure compares to a well-established test, which is 
Table 1 Level of evidence (GRADE) adapted from Guatt et al. [36] and Horvath [37]

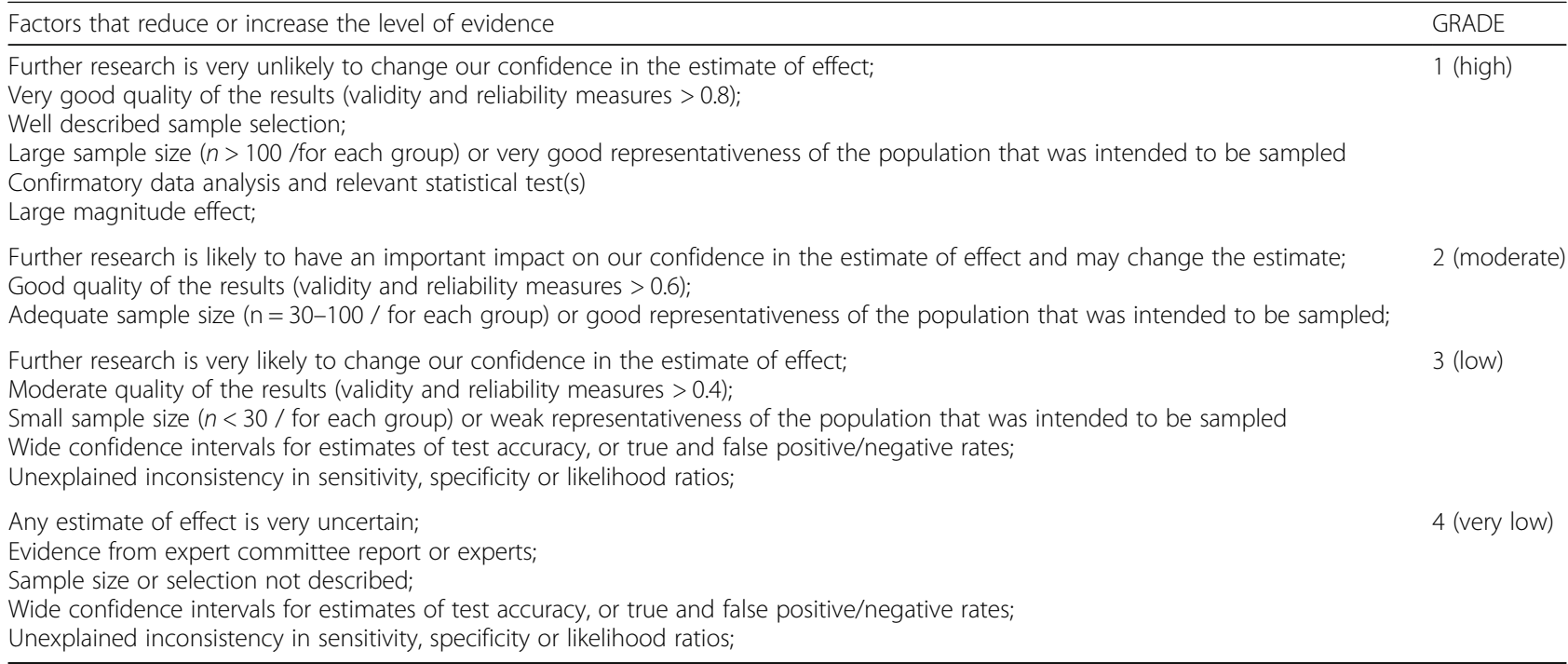

often a standardized "gold standard" test, and the evidence is obtained about the same time as the target measurement. Predictive validity is often described in terms of sensitivity and specificity. Sensitivity is the ability for a measurement to detect someone with a condition (e.g., a child with DCD). Specificity refers to the ability to correctly identify those persons without a condition (e.g., children developing typically). The required standard of sensitivity is $>80 \%$ and of specificity $>90 \%$ [3]. Construct validity is relevant to the perceived overall validity of the measurement. It is defined as the theoretical basis for using the measurement, and the methods used are often factor analysis. Known group validity examines whether a test distinguishes between a group of individuals known to have a DCD and a group who are developing typically. Discriminative validity verifies that measures or tests that should not be related are in reality not related. Convergent validity refers to the degree to which two measures of constructs that theoretically should be related are in reality related. Known group and discriminative validity and convergent validity are all considered subcategories of construct validity. Crosscultural validity applies when questionnaires have been translated into different cultures and languages. Validity can be explored by comparison of score level attributes or measurement constructs between the original and adapted versions: Does the scale work in the same way in a different population (measurement invariance and differential item functioning)? Face validity refers to the extent to which one or more individuals subjectively think that a questionnaire appears to cover the concept it purports to measure.

Reliability is the overall consistency of a measure, describing the extent to which a measure is stable when repeated under consistent conditions. First, test-retest reliability refers to the relative stability of the assessment over time, assessing the degree to which the measurement tool scores are consistent from one test administration to the next. Second, inter-rater reliability assesses the degree of agreement between two raters. Third, internal consistency assesses how well the items in the questionnaire measure the same construct. Measures of 0.80 or above are considered excellent, and the minimum acceptable value is 0.70 [40].

\section{Results}

The literature search retrieved 1907 potentially relevant publications (see Additional file 1). Of these, 1766 studies failed to meet the inclusion criteria, and 141 eligibility studies were selected. After additional searches and exclusions (Fig. 1), the final number of studies that met the inclusion criteria of our systematic review was 45 . Altogether, 11 different questionnaire-based screening tools were found, originating from 17 different countries from every continent. Six questionnaires were intended for teachers, five for parents and one for children (see Tables 2, 3 and 4).

Additional file 2 provides a summary of the characteristics of the studies included in the review. The quality of evidence, the GRADE evaluation and psychometric properties in reviewed articles can be found in Table 5 . Cross-cultural adaptations, in which reliability and/or validity was investigated in a different country from the one in which the original questionnaire was developed was examined in most of the studies $(n=26 ; 58 \%)$.

There were six tools intended for teachers' use, for children in the age range of 3-12 years. Table 2 presents the descriptive characteristics. Four observational questionnaires were for parents suitable for children aged 3.9 to 15.6 years. The descriptive characteristics of the tools completed by parents are shown in Table 3. Children's 


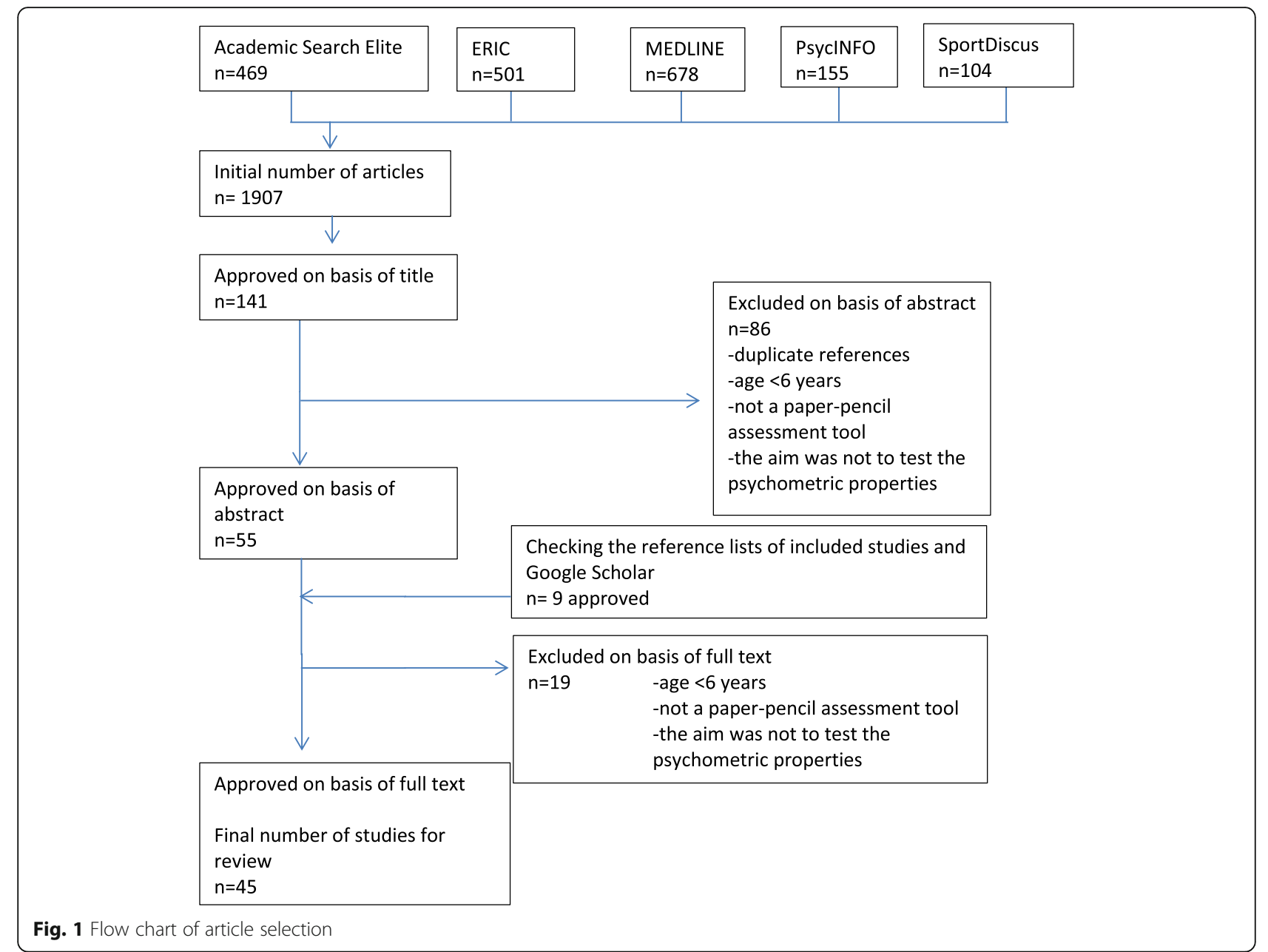

Self-Perceptions of Adequacy in and Predilection for Physical Activity (CSAPPA) for children was the only measurement in that target group, which was aimed for children in the age range of 9-16 years. Its descriptive characteristics are summarized in Table 4.

The Developmental Coordination Disorder Questionnaire (DCDQ), developed in Canada, had the most cultural adaptations in different countries and it has relatively more psychometric testing than the other tools included in this review. However, there are still some developmental needs. The inter-rater reliability and face validity has not been studied. Other cultural adaptations had MOQ-T [41, 42], MABC-2-C [25, 43-45] and TEAF [16, 46].

\section{Data synthesis}

The heterogeneity of measurement tools and study design makes comparison of screening tools very challenging. We found different kinds of samples: clinical-referred and population-based. In addition, all of the studies reviewed in this paper did not use DCD-term. But they determined children with motor coordination problems for the most part at or below the 5th or 15th percentile, which is one of the four and arguably the most important criteria of DCD [47]. Further, the studies used different measurement tools as a "gold standard" and different cut-offs to distinguish children with DCD from children developing typically; therefore, comparisons of the psychometric properties of the questionnaires are complex. Because of the difficulty of comparing the questionnaires, we recorded the advantages (strengths) and developmental needs separately from each questionnaire in Tables 2, 3 and 4. In the Additional file 2 detailed psychometric properties of the studies are described. Based on the quality evaluation (GRADE), we have confidence in those questionnaires that have been properly planned (study selection, sample, methods) and implemented carefully and of which validity and reliability criteria are acceptable. Overall, the quality of the studies was relatively good. Ten of the studies included met the highest criteria in the quality of evidence classification system (GRADE, Table 5).

\section{Synthesis of psychometric properties of the questionnaires}

Outcomes of psychometric properties represented in these studies were usability $(n=14)$, concurrent validity 
Table 2 Descriptive characteristics of observational questionnaires completed by teachers

\begin{tabular}{|c|c|c|c|c|}
\hline Measure & Country & Age range & Studies involved & Conclusions and main findings1 \\
\hline ChAS-T & Israel & $4-8 \mathrm{yrs}$ & Rosenblum [58] & $\begin{array}{l}\text { Strengths: } \\
\text {-Good item consistency and concurrent validity } \\
\text {-Distinguishes between children with and without DCD } \\
\text { To be developed: } \\
\text {-Larger samples and wider age range } \\
\text { (validity and reliability studied only in age range of 5-6.5 } \\
\text { years) } \\
\text {-Gender difference not studied } \\
\text {-No sensitivity or specificity scores } \\
\text {-Neither intra-rater nor test-retest reliability results }\end{array}$ \\
\hline Checklist & UK & $\begin{array}{l}\text { school-age } \\
\text { children }\end{array}$ & Dussart [71] & $\begin{array}{l}\text { Strength: } \\
\text {-The first screening instrument developed for teachers in the } \\
\text { normal school population } \\
\text { To be developed: } \\
\text {-Sample selection and size not described } \\
\text {-No reliability information } \\
\text {-Validity studied only superficially } \\
\text {-Many false positives }\end{array}$ \\
\hline GMRS & Netherlands & $3-7$ yrs. & Netelenbos [61] & $\begin{array}{l}\text { Strength: } \\
\text {-Good reliability } \\
\text { To be developed: } \\
\text {-No sensitivity and specificity scores }\end{array}$ \\
\hline $\begin{array}{l}M-A B C-C / \\
M-A B C-2-C\end{array}$ & UK & $\begin{array}{l}5.4-15.6 \\
\text { yrs. }\end{array}$ & $\begin{array}{l}\text { Capistrano et al. [72]; De Milander [73]; } \\
\text { Green et al. [67]; Junaid et al. [63]; Piek } \\
\text { \& Edwards [43]; Schoemaker et al. [25]; } \\
\text { Schoemaker et al. [44]; Wright et al. [45]; } \\
\text { Wright \& Sugden [74] }\end{array}$ & $\begin{array}{l}\text { Strengths: } \\
\text {-Some good test-retest reliability scores } \\
\text {-Translated in many countries } \\
\text { To be developed: } \\
\text {-Too long and time-consuming } \\
\text {-Very low sensitivity: none of the studies met the required } \\
\text { criteria } \\
\text {-Inter-rater reliability not studied }\end{array}$ \\
\hline MOQ-T & Netherlands & $5-11$ yrs. & $\begin{array}{l}\text { Asunta et al. [41]; Giofre et al. [42]; } \\
\text { Schoemaker et al. [62] }\end{array}$ & $\begin{array}{l}\text { Strengths: } \\
\text { - Good construct validity } \\
\text { - Sensitivity met the criteria } \\
\text { - Good discriminant validity and concurrent validity } \\
\text { - High internal consistency } \\
\text { - Good sample sizes } \\
\text { - Both population and clinical referred samples } \\
\text { - Fast to fill, usability good } \\
\text { To be developed: } \\
\text { - Specificity is slightly too low } \\
\text { - Inter-rater and test-retest reliability not studied }\end{array}$ \\
\hline TEAF & Canada & 6-11 yrs. & $\begin{array}{l}\text { Faught et al. [91]; Engel-Yeger et al. [16]; } \\
\text { Rosenblum \& Engel-Yeger [47] }\end{array}$ & $\begin{array}{l}\text { Strengths: } \\
\text { - Sensitivity met the criteria } \\
\text { - Predicts participation preference } \\
\text { To be developed: } \\
\text { - Specificity is slightly too low } \\
\text { - No inter-rater or test-retest reliability scores }\end{array}$ \\
\hline
\end{tabular}

Note. ChAS-T= Children Activity Scale for Teachers; GMRS= Gross Motor Rating Scale; M-ABC-C= Movement Assessment Battery for Children Checklist; M-ABC-2-C= Movement Assessment Battery for Children Checklist - Second Edition; MOQ-T= Motor Observation Questionnaire for Teachers; TEAF= Teacher Estimation of Activity Forms

${ }^{1}$ Conclusions and main findings are recapitulated by authors. Good sensitivity (>80\%), high specificity (>90\%)

$(n=31)$, predictive validity $(n=29)$, construct validity $(n=27)$, known group validity/discriminative validity $(n=30)$, convergent validity $(n=16)$, cross-cultural validity $(n=25)$, face validity $(n=5)$, internal consistency $(n=28)$, test-retest reliability $(n=12)$, and inter-rater reliability $(n=1)$. As shown in the above and in Table 4 , reliability, other than internal consistency of measures, was examined in very few studies. In summary, the inter-rater reliability and face validity were examined the least, and concurrent and discriminative validity was investigated the most. The greatest variability in terms of considering reliability and validity were the studies of Martini et al. [48] and Schoemaker et al. [44].

Convergent validity between two observational questionnaires varied from 0.16 to 0.64 , and concurrent validity between a questionnaire and a motor/screening test, correlation outcomes ranged between 0.037 and 0.76 . The good concurrent validity values were found when DCDQPL was compared to KTK-test $(r=0.73)$ and the TEAF to MABC test $(r=0.76)$. The most frequently used test to 
Table 3 Descriptive characteristics of observational questionnaires filled in by parents

\begin{tabular}{|c|c|c|c|c|}
\hline Measure & Country & Age range & Studies involved & Conclusions and main findings ${ }^{1}$ \\
\hline CAMP & Hong Kong & $5-10 \mathrm{yrs}$. & Tsang et al. $[59,87]$ & $\begin{array}{l}\text { Strengths: } \\
\text { - A promising measure } \\
\text { - Distinguishes between children with DCD and } \\
\text { TD children } \\
\text { - Good test-retest reliability } \\
\text { To be developed: } \\
\text { - Predictive validity, usability and inter-rater -reliability } \\
\text { not studied }\end{array}$ \\
\hline $\mathrm{CBCL}$ & Australia & $3.9-14.10 \mathrm{yrs}$. & Piek et al. [7] & $\begin{array}{l}\text { Strengths: } \\
\text { - Some of the items bore a relationship to motor ability } \\
\text { but they should not be used to screen DCD children } \\
\text { To be developed: } \\
\text { - Discrimination accuracy and sensitivity are poor } \\
\text { - No reliability studies done }\end{array}$ \\
\hline ChAS-P & Israel & $4-8 \mathrm{yrs}$. & Rosenblum [58] & $\begin{array}{l}\text { Strengths: } \\
\text { - Good item consistency and concurrent validity } \\
\text { - Distinguishes between children with and without DCD } \\
\text { To be developed: } \\
\text { - Small sample size } \\
\text { - validity and reliability studied only in the age range of } \\
5 \text { - } 6.5 \text { years -Gender difference not studied } \\
\text { - No sensitivity or specificity rates, neither intra-rater nor } \\
\text { inter-rater reliability results }\end{array}$ \\
\hline DCDQ & Canada & $5-15$ yrs. & $\begin{array}{l}\text { Cairney et al. [66]; Caravele et al. [75]; } \\
\text { Caravale et al. [54]; Civetta \& Hillier [76] } \\
\text { Girish et al. [77]; Green et al. [67]; } \\
\text { De Milander et al. [89]; Kennedy-Behr et al. } \\
\text { [78]; Loh et al. [79]; Martini et al. [48]; } \\
\text { Missiuna et al. [31]; Miyachi et al. [80]; }\end{array}$ & $\begin{array}{l}\text { - Most studied and evaluated questionnaire } \\
\text { - A valid clinical tool, but not for population-based } \\
\text { screening } \\
\text { To be developed: } \\
\text { - No inter-rater reliability results } \\
\text { - No face validity }\end{array}$ \\
\hline DCDDailyQ & Netherland & $5-8 \mathrm{yrs}$. & Van der Linde et al. [53] & $\begin{array}{l}\text { Strengths: } \\
\text { - Excellent discriminant validity and predictive validity } \\
\text { To be developed: } \\
\text { - No reliability results } \\
\text { - Usability descriptions and evaluation }\end{array}$ \\
\hline
\end{tabular}

Note. $\mathrm{CAMP}=$ Caregiver Assessment of Movement Participation; $\mathrm{CBCL}=$ Child Behavior Checklist; $\mathrm{ChAS}-\mathrm{P}=$ Children Activity Scales for Parents; $\mathrm{DCDQ}=$ Developmental Coordination Disorder Questionnaire

${ }^{1}$ Conclusions and main findings are recapitulated by authors. Good sensitivity (>80\%), high specificity (>90\%)

evaluate the concurrent or predictive validity with the questionnaire was MABC or MABC-2 (60\%) [49, 50]. Clearly less used were BOTM or BOTM-SF $(8,9 \%)$ [51], and MAND (4.4\%) [52]. Other measures, like KTK, and TGMD, were used under $2,3 \%$ of the cases.

Sensitivity varied in clinical referred samples between 29 and $88 \%$ and in population-based samples from 17 to $88 \%$. The specificity of the questionnaires ranged from 27 to $98 \%$ in population samples and from 19 to $95 \%$ in referred/clinic samples. Just one questionnaire, DCDDailyQ [53] reached the desired standard of predictive validity in population-based sample (sensitivity $88 \%$ and specificity 92\%; AUC .961). In clinical samples, only one measure, DCDQ-Italian [54], was adequate (sensitivity $88 \%$, specificity $96 \%$ ), but the sample size was too small for this measure to be recommended for the present purpose.

Table 4 Descriptive characteristics of approved studies completed by children

\begin{tabular}{|c|c|c|c|c|}
\hline Measure & Country & Age Range & Studies involved & Conclusions and main findings 1 \\
\hline \multicolumn{5}{|l|}{ Children } \\
\hline \multirow[t]{2}{*}{ CSAPPA } & Canada & $9-16$ yrs & Cairney et al. [24]; Hay et al. [90] & Strengths: \\
\hline & & & & $\begin{array}{l}\text {-A promising screening instrument for DCD } \\
\text {-Specificity low in population-based sample } \\
\text {-Gives important information on child's perception } \\
\text { To be developed: } \\
\text {-Reliability and usability not studied }\end{array}$ \\
\hline
\end{tabular}


Table 5 Psychometric properties of the questionnaires

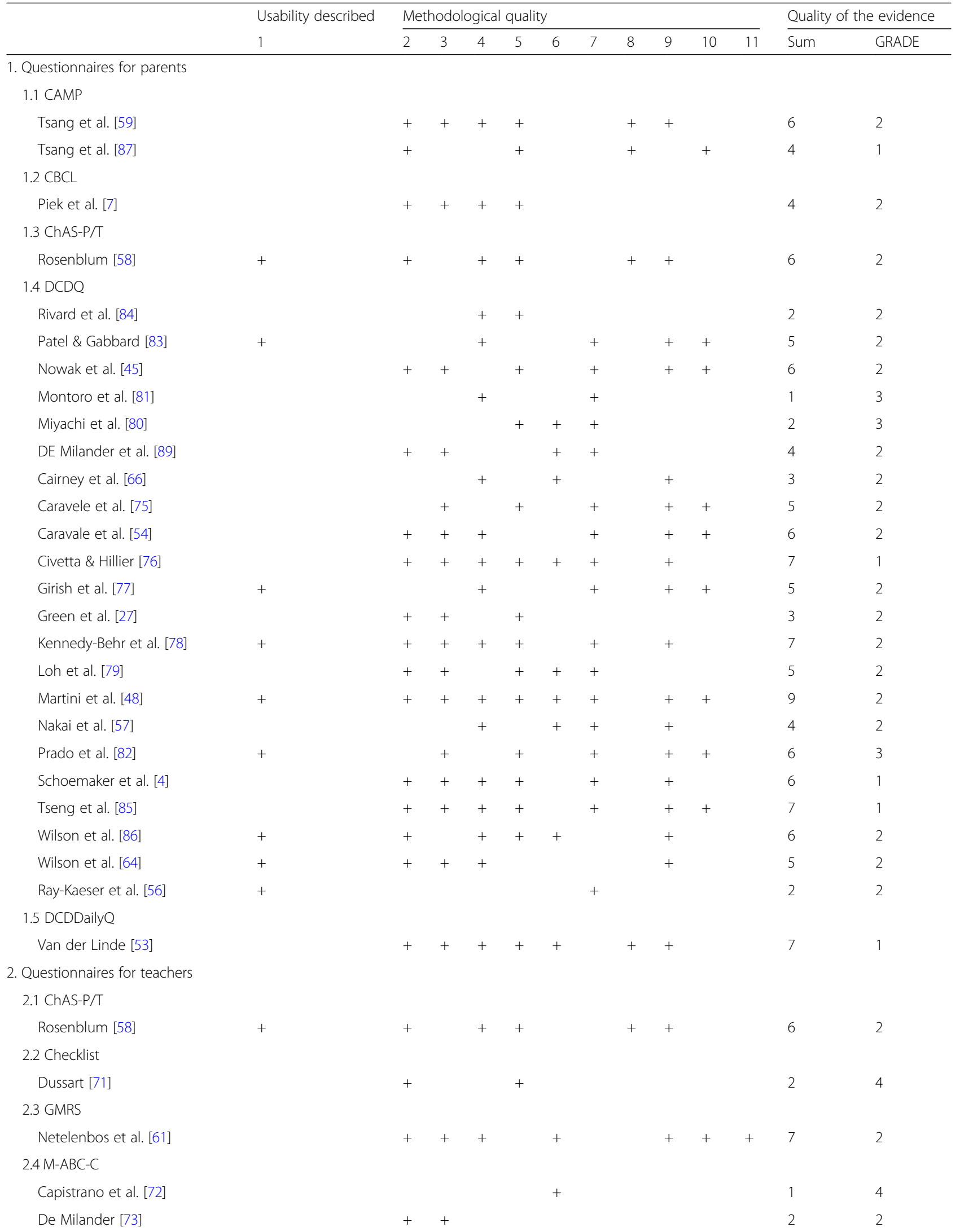


Table 5 Psychometric properties of the questionnaires (Continued)

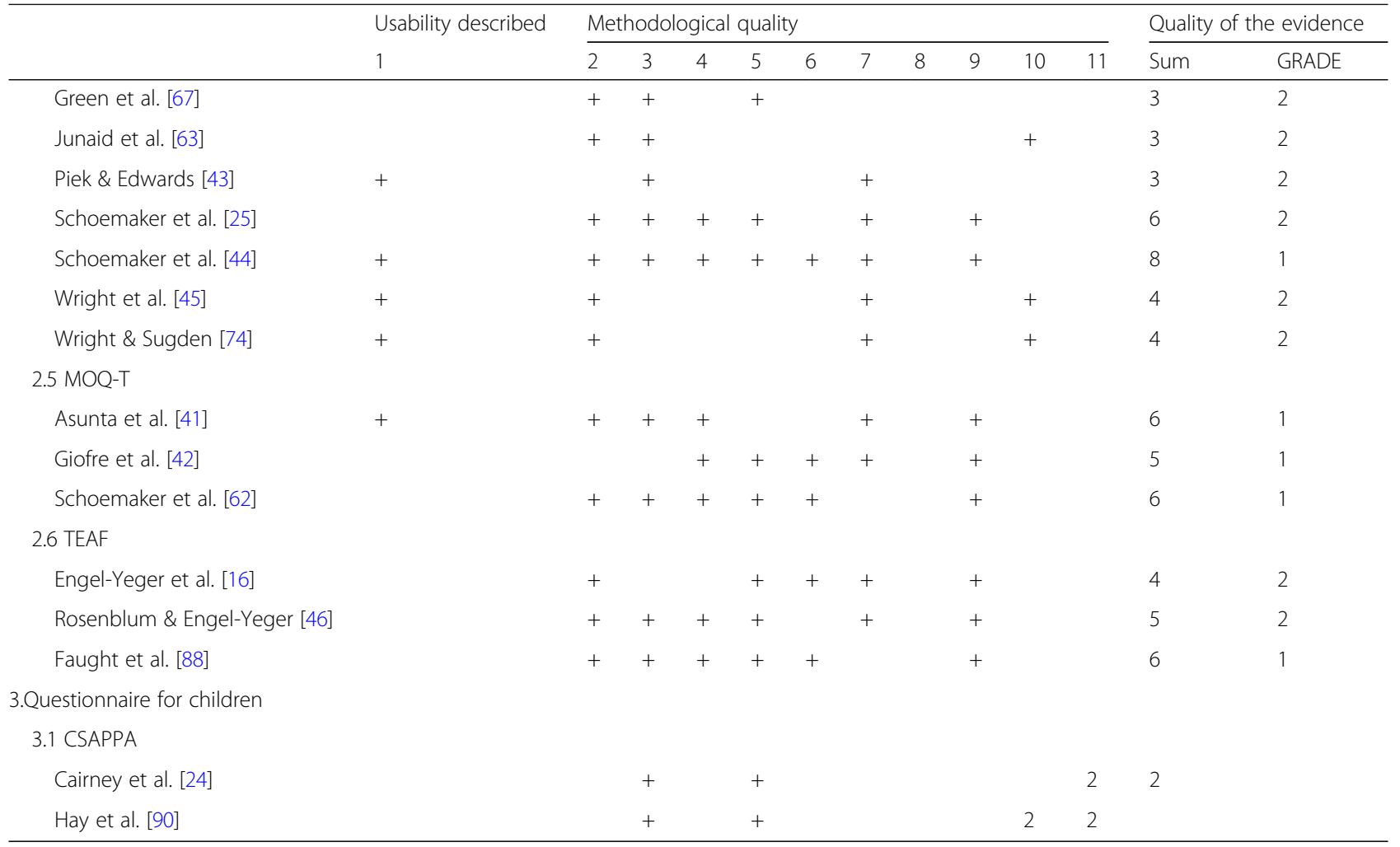

Note. 1 = usability described; $2=$ concurrent validity; $3=$ predictive validity; $4=$ construct validity; $5=$ known group validity/ discriminative validity; $6=$ convergent validity; $7=$ cross cultural validity; $8=$ face validity; $9=$ internal consistency; $10=$ test-retest reliability; $11=$ inter-rater reliability; SUM = the number of usability, validity and reliability assessment, not equivalent; GRADE criteria $(1=$ high $-4=$ low $)$

\section{Synthesis of the usability of the questionnaires}

Usability of the questionnaire was described only in $31 \%$ of the studies (see Table 5). In these studies, the most descriptions dealt with how much time evaluating requires, or how many questions / items are included in the measure. Whether users understand the questions, were explored only in a few studies. There was no study in which usability had been evaluated accurately or comprehensively. However, the ChAS-P/ $\mathrm{T}$ and MOQ-T-FI questionnaires have well described usability.

\section{Discussion}

This review evaluated 45 relevant studies and 11 observational tools for screening DCD. Overall, in many of these questionnaires, the psychometric properties and/ or feasibility was not extensively studied.

Validity evidence of a measurement tool cannot be generalized to all situations or with different attributes of population [38], therefore continuous validity and reliability evaluation of the developed methods is urgent. The translations and cross-cultural validations should be undertaken with the most stringent research design (see guidelines Beaton et al. [55]). Cognitive interviewing, which was used by Ray-Keaser et al. [56], seemed for example to be highly competent and quality approach to evaluate the cultural validity and usability of the measure.

The first step in identifying children with DCD is to be clear about the purpose of the assessment and then choose a test/tool that has been validated in that purpose [23]. Barnett [29] suggested also that selection of assessment tools to identify children with DCD should be justified and thought carefully. The selection of observational tools for children with motor difficulties will depend on their intended purpose: identification (i.e. educational settings), screening (i.e. health care), prediction, or evaluation (e.g. intervention). Many studies in this review claimed that they were appropriate for more than one purpose or in different samples. However, a measurement tool cannot be recommended if there is a lack of evidence about its psychometric properties. Therefore, it is important to be skeptical about the conclusions in some studies, because some did not have validity or reliability results that met the criteria, the sample size was too small, or the age range was too narrow [57-59]. Missiuna et al. [31] underlines also that assessors need to determine whether the level of reliability is suitable for their particular needs, for example in the particular age groups.

We recommend collecting information about the child's everyday life multiprofessionally and in different 
environments, because motor skills are often changing in diverse situations. Also in clinical practice, we would recommend using more than one observational tool to give information on motor skill difficulties in different ecological environments, this being one of the criteria in DSM-V [3] for the diagnosis of DCD.

Both reliability and validity studies should always add descriptions of the raters' background, expertise and prior training with these questionnaires. Appropriate training of raters could minimize measurement error. It is shown that the validity will improve if the observer gets sufficient information about DCD and/or the screening tool [60]. Because training and information are affecting the results, we recommend that future studies should report precisely have the assessors been trained to use the measure or given information about the motor learning problems, like DCD.

According to diagnostic criteria of DCD, motor problems affect academic achievement, leisure, and play. Based on our review for teachers there are 6 tools which could be used to evaluate this issue. Nevertheless, our study shows that in many reviewed studies teacher ratings of motor skills suffer from low concurrent validity, as had been showed previously as well [61]. However, teachers' opinions could provide further confirmation of the children's difficulties [31]. Besides, there are some high correlations with standardized test: ChAS-T and MABC $\mathrm{r}=0.75$ [58] and MOQ-T and MABC $\mathrm{r}=.57$ [62]. The physical education (PE) teachers were more able to detect motor learning problems than the classroom teachers $[43,63]$. Most of the studies in which questionnaires were intended for use by teachers, lacked information on whether the teachers also teach physical education. Unfortunately, this information was missing from most of the reviewed studies.

Parents can be used to help screening children with DCD. Parents' opinions have been found to correlate better with standardized clinical tests: e.g. concurrent validity between MABC and DCDQ $\mathrm{r}=.55$ [64] and correlation between DCDQ and KTK $r=0.726$ [65], but there has been found just moderate correlations with childrens' options [66, 67].

The concurrent and predictive validities for some assessments were calculated based on judgements by the same persons, or assessments were carried out with different standardized tests. These kind of differences and variability make exact comparisons impossible. However, the low concurrent validity that was present in almost every study may be due to a difference between the nature of the activities assessed by the observational tool in real life and the standardized motor tests such as the MABC-2 [61, 67].

Predictive validity was higher in clinic-referred samples than in population-based samples. Some studies have been attempting to overcome the low sensitivity in population-based screening by implementing two-tier referral systems [31, 68]. However, low specificity (many false positives) is not such a notable concern in the school context, where assessment and support are closely linked to each other, and where the extent of support is based on recurrent assessments. Besides, in the educational context, when support is given by classroom or PE teachers or nursery teachers, extra physical activity and support for the children identified as false positives cause no harm and do not stigmatize them. For the identified children, no further assessment is necessary if support in the educational environment is deemed to be helpful. Therefore, high sensitivity is the most important issue in educational settings. However, in healthcare screening, a large number of false positives is a major challenge, because of the cost effectiveness of providing support.

Questionnaires could be used also to give information on how motor impairments are affecting children in their daily activities and in academic learning. Therefore, observational questionnaires may be useful in clinical settings and clinic-referred samples to gain a wider picture of a child's motor ability in the school or at home. As things stand at present, none of the observational screening tools in this review could be recommended on its own for health screening of DCD. However, many of the tools can assist in the diagnosis of DCD. Multiple assessments and measurement tools are recommended to give information in different aspects of motor function; thus, it is important to develop and investigate such screening tools further. Our review reflects some limitation of the studies included. With few exceptions [26, 56, 62, 65] the study sizes were relatively small.

There are some limitations in this study as well. First, it is possible that some tools remained outside of this review, because we wanted to limit the search to school-age children. Second, our study was also restricted to literature in English, and most of the articles were published in Europe, North America, and Australia. Accordingly, some potential international publications could have been missed (see [69]: language China: [70]: language Persian). However, this review suggests that future research should focus on the validation process for the developed measures. Also, a systematic review should be carried out in the whole age range, especially in the early years and for adolescents and adults. A variety of different statistical measures were reported in this review to assess the psychometric properties. The implications or future research would be to evaluate those statistical methods used. In addition, to improve reporting quality of future studies, we recommend authors to justify the relevant statistical test(s). 


\section{Conclusion}

Many tools have been developed to help in identification and screening for motor difficulties, such as DCD. The selection of observational tools for children with motor difficulties will depend on their intended purpose: identification, screening, prediction, or evaluation. As follows, in many cases, the assessment needs to be multi-faceted and multi-professional. Overall, this study shows that there is no assessment tool, which can be used in population-based screening alone, because all those reviewed have low sensitivity or specificity or only superficially assessed reliability. In the future, psychometric property testing should be improved by addressing rater qualification and usability descriptions. In addition, the stability (test-retest reliability) and homogeneity (inter-rater reliability) should be evaluated more when assessing the psychometric properties of a questionnaire. The accurate descriptions about the usability of the questionnaires should not be forgotten either.

\section{Additional files}

Additional file 1: Search strategy. (DOCX $14 \mathrm{~kb}$ )

Additional file 2: Psychometric properties of included studies. (DOCX $57 \mathrm{~kb}$ )

\section{Abbreviations}

ADBS: The Australian Disruptive Behaviors Scale; ADHD: Attention deficit/ hyperactivity disorder; ADI-R: The Autism Diagnostic Interview-Revised Questionnaire; ADL: Everyday life skills; APA DSM-V: American Psychiatric Association, Diagnostic and Statistical Manual of Mental Disorders Diagnostic; AUC: The area under the curve; BOTM: Bruininks-Oseretsky test of Motor Proficiency; BOTMP-SF: Bruininks-Oseretsky Test of Motor Proficiency, short form; C-ABC: The Movement ABC Checklist; CAMP: Caregiver assessment of movement participation; CBCL: The child behavior checklist;

CFA: Confirmatory factor analysis; ChAS-P/T: the children activity scale for parents and for teachers; CPQ: Children participation Questionnaire; CPRC: Conners Parent Rating Scale; CSAPPA: Children's Self-Perceptions of Adequacy in and Predilection for Physical Activity; DCD: Developmental coordination disorder; DCDQ: Developmental Coordination Disorder Questionnaire; EFA: Explorative factor analysis; EYMSC: Early years movement skills checklist; GMRS: Gross Motor Rating Scale; GRADE: Grading of Recommendations Assessment, Development, and Evaluation; KBIT2: Kaufman Brief Intelligence Test-2; KTK: Körperkoordinationstest für Kinder; MABC-2 Checklist: Movement assessment battery for children checklist second edition; MABC-C: Movement assessment battery for children checklist; MAND: McCarron Assessment of Neuromuscular Development; MOQ-T: Motor observation questionnaire for teachers; PQ: The participation questionnaire; PSQ: the performance skills questionnaire; ROC: A receiver operating characteristic curve; SLI: Specific language impairment; TD: typically developing children; TEAF: The teacher estimation of activity form; TOMI: The test of motor impairment; VMl: Developmental test of visual-motor integration; WISC-III: Wechsler Intelligence Scale for Children - III

\section{Acknowledgements}

This study was a part of the developmental research project (2012-2014) for children and adolescents with mild motor limitation executed in collaboration with the Finnish CP Association, the Niilo Mäki Institute, LIKES Research Center and the University of Jyväskylä.

\section{Authors' contributions}

PA, TA, HV, and PR planned the review. PA, HV and PR performed the literature review. PA interpreted the findings and wrote the first draft. All authors participated in completing the manuscript. All authors read and approved the final manuscript.

\section{Funding}

This study has been funded by Finland's Slot Machine Association and the University of Jyväskylä. Funding bodies has no role in the design of the study and collection, analysis and interpretation of data and in writing the manuscript. This study is part of Ph.D. Thesis conducted at the Faculty of Sport and Health Sciences, University of Jyväskylä, Finland.

\section{Availability of data and materials}

All data generated or analysed during this study are included in this published article [and its supplementary information files].

\section{Ethics approval and consent to participate}

Ethical approval was obtained from the University of Jyväskylä Ethical Committee.

\section{Consent for publication}

Not applicable in this study.

\section{Competing interests}

The authors declare that they have no competing interests.

\section{Author details}

${ }^{1}$ Faculty of Sport and Health Science, University of Jyväskylä, P.O. Box 35, FI-40014 Jyväskylä, Finland. ${ }^{2}$ Faculty of Educational Sciences and Psychology, University of Jyväskylä, P.O. Box 35, Fl-40014 Jyväskylä, Finland.

Received: 14 March 2018 Accepted: 31 July 2019

Published online: 07 September 2019

\section{References}

1. Wilson PH, Ruddock S, Smits-Engelsman B, Polatajko H, Blank R. Understanding performance deficits in developmental coordination disorder: a meta-analysis of recent research. Dev Med Child Neurol. 2013;55: 217-28.

2. Missiuna C, Gaines R, Mclean J, DeLaat D, Egan M, Soucie H. Description of children identified by physicians as having developmental coordination disorder. Dev Med Child Neurol. 2008;50(11):839-44.

3. APA. Diagnostic and Statistical Manual of Mental Disorders. 5th ed. Washington, DC: American Psychiatric Association; 2013.

4. Schoemaker MM, Boudien F, Verheij NP, Wilson BN, Reinders HA, Arend DK. Evaluation of the developmental coordination disorder questionnaire as a screening instrument. Dev Med Child Neurol. 2006;48(8):668-73.

5. Piek J, Hands B, Licari M. Assessment of motor functioning in the preschool period. Neuropsychol Rev. 2012;22(4):402-13.

6. Cairney J, Rigoli D, Piek J. Developmental coordination disorder and internalizing problems in children: the environmental stress hypothesis elaborated. Dev Rev. 2013;33(3):224-38.

7. Piek JP, Barrett NC, Dyck MJ, Reiersen AM. Can the child behavior checklist be used to screen for motor impairment? Dev Med Child Neurol. 2010;52(2): 200-4.

8. Rivilis I, Hay J, Cairney J, Klentrou P, Liu J, Faught J. Physical activity and fitness in children with developmental coordination disorder: a systematic review. Res Dev Disabil. 2011;32(3):894-910.

9. Dewey D, Bernier FP. The concept of atypical brain development in developmental coordination disorder (DCD)—a new look. Curr Dev Disorders Rep. 2016:3(2):161-9.

10. Rasmussen P, Gillberg C. Natural outcome of ADHD with developmental coordination disorder at age 22 years: a controlled, longitudinal, communitybased study. J Am Acad Child Adolesc Psychiatry. 2000;39:1424-31.

11. Missiuna C, Cairney J, Pollock N, Campbell W, Russell DJ, Macdonald K, Cousins M. Psychological distress in children with developmental coordination disorder and attention-deficit hyperactivity disorder. Res Dev Disabil. 2014;35(5):1198-207.

12. Biotteau M, Chaix Y, Albaret JM. What do we really know about motor learning in children with developmental coordination disorder? Curr Dev Disorders Rep. 2016;3(2):152-60.

13. Caeyenberghs K, Taymans T, Wilson PH, Vanderstraeten G, Hosseini $H_{\text {, }}$ Waelvelde $\mathrm{H}$. Neural signature of developmental coordination disorder in 
the structural connectome independent of comorbid autism. Dev Sci. 2016; 19(4):599-612

14. Sumner E, Leonard HC, Hill EL. Overlapping phenotypes in autism spectrum disorder and developmental coordination disorder: a cross-syndrome comparison of motor and social skills. J Autism Dev Disord. 2016;46(8):2609-20.

15. Barnett AL. Motor assessment in developmental coordination disorder: from identification to intervention. Intl J Disabil Dev Educ. 2008;55(2):113-29.

16. Engel-Yeger B, Hanna-Kassis A, Rosenblum S. Can gymnastic teacher predict leisure activity preference among children with developmental coordination disorders (DCD)? Res Dev Disabil. 2012;33(4):1006-13.

17. Peersman W, Carton W, Cambier D, De Maeseneer J, van Waelvelde H. Psychometric properties of a motor skill checklist for 3- to 5-year-old children. Child Care Health Dev. 2012;38(3):350-7.

18. Rivard LM, Missiuna C, Hanna S, Wishart L. Understanding teachers' perceptions of the motor difficulties of children with developmental coordination disorder (DCD). Br J Educ Psychol. 2007;77(3):633-48.

19. Hatakenaka Y, Kotani H, Yasumitsu-Lovell K, Suzuki K, Fernell E, Gillberg C. Infant motor delay and early symptomatic syndromes eliciting neurodevelopmental clinical examinations in Japan. Pediatr Neurol. 2016;54:55-63.

20. van den Heuvel M, Jansen DE, Reijneveld SA, Flapper BC, Smits-Engelsman $B C$. Identification of emotional and behavioral problems by teachers in children with developmental coordination disorder in the school community. Res Dev Disabil. 2016;51:40-8.

21. The World Health Organization quality of life assessment (WHOQOL): position paper from the World Health Organization. Soc Sci Med. 1995; 41(10): 1403-1409.

22. Rudd J, Butson ML, Barnett L, Farrow D, Berry J, Borkoles E, Polman R. A holistic measurement model of movement competency in children. J Sports Sci Med. 2016;34(5):477-85.

23. Cools W, De Martelaer K, Samaey C, Andries C. Movement skill assessment of typically developing preschool children: a review of seven movement skill assessment tools. J Sports Sci Med. 2009:8(2):154

24. Cairney J, Veidhuizen S, Kurdyak P, Missiuna C, Faught BE, Hay J. Evaluating the CSAPPA subscales as potential screening instruments for developmental coordination disorder. Arch Dis Child. 2007;92(11):987-91. https://doi.org/1 0.1136/adc.2006.115097.

25. Schoemaker MM, Smits-Engelsman B, Jongmans MJ. Psychometric properties of the movement assessment battery for children-checklist as a screening instrument for children with a developmental co-ordination disorder. Br J Educ Psychol. 2003;73(3):425-41.

26. Schoemaker M, Flapper B, Verheij N, Wilson B, Reinders-Messelink H, de Kloet A. Evaluation of the developmental coordination disorder questionnaire as a screening instrument. Dev Med Child Neurol. 2006:48:668-73.

27. Green D, Wilson BN. The importance of parent and child opinion in detecting change in movement capabilities. Can J Occup Ther. 2008;75(4):208-19.

28. Gwynne K, Blick B, Hughes L. Use of an occupational therapy motor performance checklist by a school health service: a pilot study. J Paediatr Child Health. 1996;32(5):386-90.

29. Barnett L, Van Beurden E, Morgan P, Brooks L, Beard J. Does childhood motor skill proficiency predict adolescent fitness? Med Sci Sports Exerc. 2008;40(12):2137.

30. Portney LG, Watkins MP. Foundations of clinical research: applications to practice. New Jersy: Pearson Education; 2009. p. 3.

31. Missiuna C, Cairney J, Pollock N, Russell D, Macdonald K, Cousins M, et al. A staged approach for identifying children with developmental coordination disorder from the population. Res Dev Disabil. 2011;32(2):549-59.

32. Schoemaker MM \& Wilson B. In J. Cairney (Ed.). Developmental coordination disorder and its consequences. 2015; 169-191. University of Toronto Press.

33. EACD, European Academy of Childhood Disability. EACD Recommendations, long version. Definition, diagnosis, assessment and intervention of Developmental Coordination Disorder. 2011; 1-115.

34. Rutter D, Francis J, Coren E, Fisher M. SCIE systematic research reviews: guidelines. London: Social Care Institute for Excellence; 2010.

35. GRADE working group. http://www.gradeworkinggroup.org/

36. Guyatt $\mathrm{GH}$, Oxman AD, Schünemann HJ, Tugwell P, Knottnerus A. GRADE guidelines: a new series of articles in the journal of clinical epidemiology. J Clin Epidemiol. 2011;64(4):380-2.

37. Horvath A. Grading quality of evidence and strength of recommendations for diagnostic tests and strategies. Clin Chem. 2009;55(5):853-5.

38. Yun J, Ulrich DA. Estimating measurement validity: a tutorial. APAQ. 2002; 19(1):32-47
39. Messick S. Validity of psychological assessment: validation of inferences from persons' responses and performances as scientific inquiry into score meaning. Am Psychol. 1995;50(9):741.

40. Terwee CB, Bot SDM, Boer MR, Van der Windt DA, Knoll DL, Dekker J, et al. Quality criteria were proposed for measurement properties of health status questionnaires. J Clin Epidemiol. 2007;60:34-42.

41. Asunta P, Viholainen $H$, Ahonen T, Cantell M, Westerholm J, Schoemaker MM, Rintala P. Reliability and validity of the Finnish version of the motor observation questionnaire for teachers. Hum Movement Sci. 2017;53:63-71.

42. Giofre D, Cornoldi C, Schoemaker MM. Identifying developmental coordination disorder: MOQ-T validity as a fast screening instrument based on teachers' ratings and its relationship with praxic and visuospatial working memory deficits. Res Dev Disabil. 2014;35(12):3518-25.

43. Piek JP, Edwards K. The identification of children with developmental coordination disorder by class and physical education teachers. $\mathrm{Br} J$ Educ Psychol. 1997;67(1):55-67.

44. Schoemaker MM, Niemeijer AS, Flapper BC, Smits-Engelsman BC. Validity and reliability of the movement assessment battery for children-2 checklist for children with and without motor impairments. Dev Med Child Neurol. 2012;54(4):368-75

45. Wright HC, Sugden DA, Ng R, Tan J. Identification of children with movement problems in Singapore: usefulness of the movement $A B C$ checklist. APAQ. 1994;11(2):150-7.

46. Rosenblum S, Engel-Yeger B. Hypo-activity screening in school setting; examining reliability and validity of the teacher estimation of activity form (Teaf). Occup Ther Int. 2015;22(2):85-93.

47. Cairney J, Veldhuizen S. Is developmental coordination disorder a fundamental cause of inactivity and poor health-related fitness in children? Dev Med Child Neurol. 2013;55(s4):55-8.

48. Martini R, St-Pierre M, Wilson BN. French Canadian cross-cultural adaptation of the developmental coordination disorder questionnaire '07: DCDQ-FC. Can J Occup Ther. 2011;78(5):318-27.

49. Henderson SE, Sugden DA, Barnett AL. Movement assessment battery for children. London: Psychological Corporation; 1992.

50. Henderson SE, Sugden DA, Barnett AL. Movement Assessment Battery for Children-2. 2nd ed. London: Harcourt Assessment; 2007.

51. Bruininks R, Bruininks B. Bruininks Oseretsky Test of Motor Proficiency. 2nd ed. Minneapolis MN: NCS Pearson; 2005.

52. McCarron LT. MAND McCarron assessment of neuromuscular development, fine and gross motor abilities (rev. ed.): Common Market Press, Dallas, TX; 1982.

53. van Der Linde BW, van Netten JJ, Otten BE, Postema K, Geuze RH, Schoemaker MM. Psychometric properties of the DCDDaily-Q: a new parental questionnaire on children's performance in activities of daily living. Res Dev Disabil. 2014;35(7):1711-9.

54. Caravale B, Baldi S, Capone L, Presaghi F, Balottin U, Zoppello M. Psychometric properties of the Italian version of the developmental coordination disorder questionnaire (DCDQ-Italian). Res Dev Disabil. 2015;36:543-50.

55. Beaton DE, Bombardier C, Guillemin F, Ferraz MB. Guidelines for the process of cross-cultural adaptation of self-report measures. Spine. 2000; 25(24):3186-91.

56. Ray-Kaeser S, Satink T, Andresen M, Martini R, Thommen E, Bertrand AM European-French cross-cultural adaptation of the developmental coordination disorder questionnaire and pretest in French-speaking Switzerland. Phys Occup Ther Pediatr. 2015;35(2):132-46.

57. Nakai A, Miyachi T, Okada R, Tani I, Nakajima S, Onishi M, et al. Evaluation of the japanese version of the developmental coordination disorder questionnaire as a screening tool for clumsiness of japanese children. Res Dev Disabil. 2011;32(5):1615-22.

58. Rosenblum S. The development and standardization of the children activity scales (ChAS-P/T) for the early identification of children with developmental coordination disorders. Child Care Health Dev. 2006;32(6):619-32.

59. Tsang KL, Stagnitti K, Lo SK. Screening children with developmental coordination disorder: the development of the caregiver assessment of movement participation. J Child Health Care. 2010;39(3):232-48.

60. Chambers ME, Sugden D. The identification and assessment of young children with movement difficulties. Int J Early Years Educ. 2002;10(3):157.

61. Netelenbos JB. Teachers' ratings of gross motor skills suffer from low concurrent validity. Hum Movement Sci. 2005;24(1):116-37. https://doi.org/1 0.1016/j.humov.2005.02.001.

62. Schoemaker MM, Flapper BCT, Reinders-Messelink $H$, Kloet AD. Validity of the motor observation questionnaire for teachers as a screening instrument 
for children at risk for developmental coordination disorder. Hum Movement Sci. 2008;27(2):190-9.

63. Junaid K, Harris SR, Fulmer KA, Carswell A. Teachers' use of the MABC checklist to identify children with motor coordination difficulties. Pediatr Phys Ther. 2000;12(4):158-63.

64. Wilson BN, Crawford SG, Green D, Roberts G, Aylott A, Kaplan BJ. Psychometric properties of the revised developmental coordination disorder questionnaire. Phys Occup Ther Pediatr. 2009;29(2):184-204

65. Nowak A. Cross-cultural adaptation of the developmental coordination disorder questionnaire (DCDQ'07) for the population of polish children. Biomed Hum Kinet. 2016;8(1):17-23.

66. Cairney J, Missiuna C, Veldhuizen S, Wilson B. Evaluation of the psychometric properties of the developmental coordination disorder questionnaire for parents (DCD-Q): results from a community based study of school-aged children. Hum Movement Sci. 2008;27(6):932-40.

67. Green D, Bishop T, Wilson BN, Crawford S, Hooper R, Kaplan B, Baird G. Is questionnaire-based screening part of the solution to waiting lists for children with developmental coordination disorder? Br J Occup Ther. 2005; 68(1):2-10.

68. Gwynne K, Blick B. Motor performance checklist for 5-year-olds: a tool for identifying children at risk of developmental co-ordination disorder. J Paediatr Child Health. 2004;40(7):369-73.

69. Hua J, Wu ZC, Gu GX, Meng W. Assessment on the application of 'Movement Assessment Battery'for Children. Zhonghua Liu Xing Bing Xue Za Zhi. 2012;33(10):1010-5 (abstract English).

70. Salehi H, Zarezadeh M, Salek B. Validity and Reliability of the Persian Version of Motor Observation Questionnaire for Teachers (PMOQ-T). Iran J Psychiatry. 2012;18(3):211-9 (abstract English).

71. Dussart G. Identifying the clumsy child in school: an exploratory study. Br J Special Educ. 1994;21(2):81-6.

72. Capistrano R, Ferrari EP, Souza LPD, Beltrame TS, Cardoso FL. Concurrent validation of the MABC-2 motor tests and $\mathrm{MABC}-2$ checklist according to the developmental coordination disorder questionnaire-br. Motriz: J Phys Ed. 2015;21(1):100-6.

73. De Milander M, Coetzee FF, Venter A. Teachers' ability to identify children with developmental coordination disorder. AJPHES. 2016;22(Issue-41):990-1005.

74. Wright HC, Sugden DA. A two-step procedure for the identification of children with developmental co-ordination disorder in Singapore. Dev Med Child Neurology. 1996;38(12):1099-105.

75. Caravale B, Baldi S, Gasparini C, Wilson BN. Cross-cultural adaptation, reliability and predictive validity of the italian version of developmental coordination disorder questionnaire (DCDQ). Eur J Paediatr Neurol. 2014; 18(3):267-72.

76. Civetta LR, Hillier SL. The developmental coordination disorder questionnaire and movement assessment battery for children as a diagnostic method in australian children. Pediatr Phys Ther. 2008;20(1): 39-46.

77. Girish S, Raja K, Kamath A. Translation of revised version of developmental coordination disorder questionnaire (DCDQ'07) into Kannada-results of validation. APDRJ. 2015;26(4):82-100.

78. Kennedy-Behr A, Wilson BN, Rodger S, Mickan S. Cross-cultural adaptation of the developmental coordination disorder questionnaire 2007 for Germanspeaking countries: DCDQ-G. Neuropediatrics. 2013:44(5):245-51.

79. Loh P, Piek J, Barrett NC. The use of the developmental coordination disorder questionnaire in Australian children. APAQ. 2009;26(1):38-53.

80. Miyachi T, Nakai A, Tani I, Ohnishi M, Nakajima S, Tsuchiya KI, Tsujii M. Evaluation of motor coordination in boys with high-functioning pervasive developmental disorder using the Japanese version of the developmental coordination disorder questionnaire. J Dev Phys Disabil. 2014;26(4):403-13.

81. Montoro APPN, Capistrano R, Ferrari EP, da Silva RM, Cardoso FL, Beltrame TS. Concurrent validation of the MABC-2 and developmental coordination disorder questionnaire-BR. J Hum Growth Dev. 2016;26(1):74-80.

82. Prado MSS, Magalhães LC, Wilson BN. Cross-cultural adaptation of the developmental coordination disorder questionnaire for brazilian children. Braz J Phys Ther. 2009;13(3):236-43.

83. Patel P, Gabbard C. Adaptation and preliminary testing of the developmental coordination disorder questionnaire (DCDQ) for children in India. Phys Occup Ther Pediatr. 2017;37(2):170-82.

84. Rivard L, Missiuna C, McCauley D, Cairney J. Descriptive and factor analysis of the developmental coordination disorder questionnaire (DCDQ '07) in a population-based sample of children with and without developmental coordination disorder. Child Care Health Dev. 2014;40(1):42-9.

85. Tseng M, Fu C, Wilson BN, Hu F. Psychometric properties of a Chinese version of the developmental coordination disorder questionnaire in community-based children. Res Dev Disabil. 2010;31(1):33-4.

86. Wilson BN, Kaplan BJ, Crawford SG, Campbell A, Dewey D. Reliability and validity of a parent questionnaire on childhood motor skills. Am J Occup Ther. 2000;54(5):484-93.

87. Tsang KL, Bond T, Lo SK. Psychometric properties of the caregiver assessment of movement participation scale for screening children with development coordination disorders. Intl J Disabil Dev Educ. 2010a;57(4): 383-402.

88. Faught BE, Cairney J, Hay J, Veldhuizen S, Missiuna C, Spironello CA. Screening for motor coordination challenges in children using teacher ratings of physical ability and activity. Hum Movement Sci. 2008:27(2):177-89.

89. De Milander M, Coetzee FF, Venter A. The ability of parents to identify Grade 1-learners with developmental coordination disorder at home. S Afr J Res Sport Ph. 2015;37(3):55-68.

90. Hay JA, Hawes R, Faught BE. Evaluation of a screening instrument for developmental coordination disorder. J Adolesc Health. 2004;34(4):308-13.

91. Doderer L, Miyahara M. Critical triangulation of a movement test, questionnaires, and observational assessment for children with DCD. Int J Ther Rehabil. 2013;20(9):435-42.

\section{Publisher's Note}

Springer Nature remains neutral with regard to jurisdictional claims in published maps and institutional affiliations.
Ready to submit your research? Choose BMC and benefit from:

- fast, convenient online submission

- thorough peer review by experienced researchers in your field

- rapid publication on acceptance

- support for research data, including large and complex data types

- gold Open Access which fosters wider collaboration and increased citations

- maximum visibility for your research: over $100 \mathrm{M}$ website views per year

At BMC, research is always in progress.

Learn more biomedcentral.com/submissions 